\title{
5
}

\section{Role of Texture Analysis and Radiomics in Prediction of Bone Marrow Infiltration in Patients with Lymphoma}

\author{
Mahmoud A. Kenawy ${ }^{1 *}$, Magdy M. Khalil ${ }^{2}$, Mahmoud H. Abdelgawad ${ }^{1}$, \\ H. H. El-Bahnasawy ${ }^{1}$ \\ 1 Biophysics Branch, Department of Physics, Faculty of Science, Al-Azhar University, Nasr City 11884, \\ Cairo, Egypt. \\ ${ }^{2}$ Department of Physics, Faculty of Science, Helwan University, Ain Helwan 11795, Cairo, Egypt.
}

\begin{abstract}
$\mathbf{T}$ O EXPLORE the ability of image-based parameters with texture parameters in the differentiation of bone marrow infiltration by positron emission tomography computed tomography (PET/CT).We retrospectively evaluated the baseline PET/CT scan of 44 patients with histopathological proven lymphoma. Seventy-three of features were extracted using CGITA software and statistical analysis were carried out on SPSS program. Result of spearman correlation analysis revealed a strong positive correlation between conventional PET metrics and texture features (19 significant association features) and inverse correlation was found (one parameter significant association feature). Area under the curve and p-value of receive operating characteristics showed that (HILRE (4-bin), HILZE (64-bin), LRE (64-bin), LZE (64-bin), max spectrum (8-bin), busyness (64-bin), code similarity (32-bin \& 64-bin)) were significant discriminator of bone marrow infiltration among other features (AUC>0.682, $\mathrm{p}<0.05$ ). Univariate analyses of texture features showed that code similarity and LRE (both 64 bin) has significant prediction to BMI. Multivariate analyses revealed that LRE (64 bin) $p=0.031$; odds ratio: $1.022 ; 95 \% \mathrm{CI}, 1.002-1.043$ ) were independent variables for bone marrow infiltration. The study concludes that a significant association emerged between PET features and bone marrow infiltration in lymphoma. Texture analysis on PET/CT shows potential to differentiate between bone marrow infiltration in patients with Lymphoma.
\end{abstract}

Keywords: Bone marrow; Fluorodeoxyglucose F-18; Image texture; Lymphoma; Positron emission tomography.

\section{Introduction}

Lymphoma is a heterogeneous type of disease that arises from the constituent cells of the immune system or from their precursors that arise in lymph nodes close to any organ or tissue in the body. Therefore, all organ systems may be involved at some stage of the disease including the central nervous system, head and neck, thorax, abdomen, gonads, and bone ${ }^{1,2}$.

Imaging modalities have a fundamental role in the staging of lymphomas. CT is the most often used imaging modality for staging malignant lymphoma due to the fact of its massive availability and enormously low cost ${ }^{3}$ but sometimes CT impedes identification of disease in normal-sized organs and detection of lesions which have poor contrast with the surrounding tissues. Another reason is that CT is not effective in diagnosis lymphoma being not reliable in the detection of bone marrow disease, which, if present, by definition indicates stage IV disease ${ }^{4}$.

${ }^{18} \mathrm{~F}-\mathrm{FDG}$ PET/CT has an impact on the assessment of both newly diagnosed, prognosed and previously treated patients with lymphoma and also alters the initial clinical staging of this disease ${ }^{5,6}$. Incidence of marrow disease varies with histological subtype of lymphoma. Bone marrow biopsy is fundamental in identifying marrow involvement for therapy purpose ${ }^{7}$. Marrow diseases can be diagnosed with ${ }^{18} \mathrm{~F}-\mathrm{FDG}$ PET/CT scan and the visual interpretation of marrow FDG uptake throughout whole-body. PET scans can efficiently determine marrow disease status in a high proportion of lymphoma patients. Therefore, PET has the potential to reduce the guide for staging marrow biopsy ${ }^{8}$. However, interobserver variability remain a limiting factor in qualitative image analysis.

A high level of concordance has been observed between the sites of focal ${ }^{18} \mathrm{~F}$ - FDG uptake in the bone marrow and bone marrow biopsy. In fact, PET scans have demonstrated a high negative predictive value to exclude bone marrow involvement. This holds particularly true for early stage lymphoma and may even obviate the need for bone marrow biopsy in this group ${ }^{9-11}$.

This result if confirmed will provide two identical methods for diagnosis of lymphomas. However, the use of ${ }^{18} \mathrm{~F}$-FDG PET/CT in the sole assessment of the bone marrow and whether it can replace bone marrow biopsy is still a topic of debate and thus investigation with PET/CT scan through image

*Corresponding author Email: mahmoudbiophysics@yahoo.com

Received 6/9/2020 ; accepted 15/9/2020 
analysis is potentially advised. ${ }^{3}$. However, it could be useful to strength the diagnostic power of PET imaging through sophisticated data analysis using the recent advances in PET texture analysis.

The aim of this study was to explore whether the textural features able to provide alternative or addition diagnostic information of bone marrow infiltration in patients diagnosed with lymphoma using ${ }^{18} \mathrm{~F}$-FDG PET/CT.

\section{Material and Methods}

\section{Patients}

A total of 44 patients $(56.8 \%$ Male) whose histopathology proven and diagnosed with lymphoma were retrospectively retrieved from our data records. The retrospective nature of the study has obviated the need to ask for patient consent. A committee ethical approval was obtained to perform the study. The inclusion criteria were those patients who had not prior treatment before ${ }^{18} \mathrm{~F}-\mathrm{FDG}$ initial diagnosis. Bone marrow involvement were confirmed by two clinical observers who have 5 and 10 years of experience in reading and interpretation of ${ }^{18} \mathrm{~F}$-FDG PET/CT. The number of patients who had bone marrow infiltration was $18(41 \%)$ as agreed by the two observers.

\section{PET/CT Examination}

Patients were fasted $6 \mathrm{~h}$ and had serum glucose concentration $<200 \mathrm{mg} / \mathrm{dl}$ before the intravenous injection of $185-370 \mathrm{MBq}$ of ${ }^{18} \mathrm{~F}-\mathrm{FDG}$ (4 MBq/kg or $0.1 \mathrm{mCi} / \mathrm{kg}$ of body weight). ${ }^{18} \mathrm{~F}-\mathrm{FDG} \mathrm{PET} / \mathrm{CT}$ was performed using a Gemini 64 LXL PT/CT scanner (Philips Medical Systems) in 7 patients, 5 patients performed on Gemini 64 TF TOF PT/CT (Philips Healthcare) whereas the remaining 32 patients were imaged on a Biograph $20 \mathrm{mCT}$ scanner (Siemens Medical Solutions). During imaging process, patients were in supine position with both arms extended in the cranial direction and breathing quietly. PET images were obtained at 2 $\mathrm{min} / \mathrm{bed}$ frame for the patient less than $60 \mathrm{~kg}, 2.5$ $\mathrm{min} / \mathrm{bed}$ frame for the patients less than $90 \mathrm{~kg}$ and 3 $\mathrm{min} /$ bed frame for the patents up to $90 \mathrm{~kg}$. PET images were reconstructed using CT-based attenuation correction by an ordered-subset expectation maximization iterative reconstruction algorithm (OSEM) with a slice thickness of $3 \mathrm{~mm}$, and a pitch of $4 \mathrm{~mm}$ for the Biograph $\mathrm{mCT}$; and slice thickness of $4 \mathrm{~mm}$, and a pitch of $4 \mathrm{~mm}$ for the both Philips machines.

\section{Feature extraction}

All images were transferred to a personal computer that contained Chang-Gung Image Texture Analysis (CGITA) toolbox version 1.4. The PET images were then imported into (CGITA) toolbox. CGITA is an open-source software code with a graphical user interface for texture analysis running on MATLAB (Math Works Inc., version 2015a) ${ }^{12}$.

Table 1: Clinical characteristics and acquisition parameters of the study population.

\begin{tabular}{|c|c|c|c|}
\hline \multirow{13}{*}{ 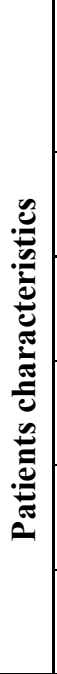 } & \multirow{3}{*}{ Age } & Median & 47 year \\
\hline & & Mean & 49.2 year \\
\hline & & Range & 19-88 year \\
\hline & \multirow{2}{*}{ Sex } & Male & 25 \\
\hline & & Female & 19 \\
\hline & \multirow{2}{*}{ Weight } & Median & $73.5(\mathrm{~kg})$ \\
\hline & & Range & $50-110(\mathrm{~kg})$ \\
\hline & \multirow{2}{*}{ Blood glucose } & Median & $102.5(\mathrm{mg} / \mathrm{dl})$ \\
\hline & & Range & $70-135(\mathrm{mg} / \mathrm{dl})$ \\
\hline & \multirow{2}{*}{$\begin{array}{c}\text { Type of } \\
\text { lymphoma }\end{array}$} & $\mathrm{HL}$ & 30 \\
\hline & & NHL & 14 \\
\hline & \multirow{2}{*}{$\begin{array}{c}\text { Bone marrow } \\
\text { invasion by PET }\end{array}$} & Yes & 18 \\
\hline & & No & 26 \\
\hline \multirow{6}{*}{ 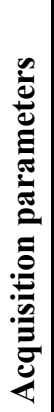 } & \multirow{2}{*}{$\begin{array}{c}\text { Injected } \\
\text { radioactivity } \\
\left({ }^{18} \text { F-FDG) }\right.\end{array}$} & Median & $277.5 \mathrm{MBq}$ \\
\hline & & Range & $185-370 \mathrm{MBq}$ \\
\hline & \multirow{2}{*}{$\begin{array}{c}{ }^{18} \text { F-FDG uptake } \\
\text { time }\end{array}$} & Median & $55 \mathrm{~min}$ \\
\hline & & Range & $50-60 \mathrm{~min}$ \\
\hline & $\begin{array}{c}\text { Time per bed } \\
\text { position }\end{array}$ & \multicolumn{2}{|c|}{ 2-3 min } \\
\hline & Scan time range & \multicolumn{2}{|c|}{$16-27 \mathrm{~min}$} \\
\hline
\end{tabular}

PET images were manually or semi-automatic contoured with an iso-contour threshold was set to an absolute SUV value of 3.0 as previously described ${ }^{13}$.

All scans were analyzed by senior residents independently (more than 5-year experience) and were interpreted by a senior radiologist (10 years of experience). The second step consisted of resampling or interpolating the non-cubic voxel grids into cubic voxels $(2 \mathrm{~mm})$ and performing quantization (also called discretization, down sampling or resampling) of the original intensities into a discrete set of values between the minimum and maximum within the VOI. This number determines the size of the matrices from which the different texture features will be subsequently calculated.

A bin number of 4, 8, 16, 32 and 64 was employed in this study. Third, a total of seventy-three radiomics parameters were extracted from images including texture features and conventional PET metrics such as SUVmax, SUVmean, SUVpeak and TLG were extracted for each VOI. SUVpeak was defined as the mean SUV within 1-cm sphere centered on the maximum pixel ${ }^{14}$. 


\section{Texture analysis}

Texture analysis includes first, second, and higher order texture methods as well as various transformbased methods. The most "direct" features are those based simply on intensity values within a region of interest (ROI). Texture features in the lymphoma is based on the same parent matrices that are utilized in tumor sites. Examples of these include the graylevel co-occurrence matrix (GLCM), the gray level run length matrix (GLRLM), the neighborhood intensity difference matrix (NIDM), neighborhood gray-level dependence matrix (NGLDM), and the intensity size-zone matrix (ISZM) ${ }^{15-17}$.

In this study, the derived features from voxels of the segmented PET images can be grouped into different categories. First-order features were derived from the histogram of voxel intensities (SUVmean, SUVmax, skewness, kurtosis, etc.). Second-order textural features were based on matrices that contained information about the regional spatial arrangement of the voxels such as their homogeneity, contrast, and coarseness simulating the human perception of the image. Higher-order features such as Grey-level run-length features focused on local collinear voxels with the same grey level. Table I in supplement material describes the extracted features using CGITA toolbox from the segmented images.

\section{Statistical Analysis}

Spearman rank correlations between all extracted features were calculated and displayed via a heatmap (Fig.1). Spearman correlation coefficient (rho) was used to evaluate the pairwise relationships between SUVmean, SUVmax, SUVpeak, TLG, surface area, and the derived 73 texture features including all bin sizes. Since not all the parameters contributed to differentiating lymphoma disease, we resorted to the results of operation characteristic curve at each bin size and to test the diagnostic potential of the quantitative indices including the conventional metrics as well as texture features in discrimination of bone marrow invasion 18, 19. A heatmap (with Pearson's correlation coefficient values) was constructed to observe correlations among the detected constituents between each feature which has a strong or moderate correlation coefficient resulted from the Spearman test for different bin size. Receive operation characteristic curves (ROC curve) were used to select the most discriminative parameters in each bin size (Fig. 2).

Specificity and sensitivity (including 95\% confidence intervals [CIs]) for each of the investigated parameters were also derived and calculated. The Youden index is the vertical distance between the 45-degree line and the point on the ROC curve. ROC data analysis was performed. Binary logistic regression test was used to express results of univariate then multivariate analysis for those features of high AUC derived from ROC data plots. All statistical analysis was performed using SPSS program (IBM Corp, Armonk, New York, version 23). A $p$-value of $<0.05$ was defined as statistically significant. No adjustment for multiple comparisons was made in this analysis.

\section{Result}

\section{Patients}

The general characteristics of the study participants are shown in Table 1. There were 25 men (mean age, 53.4 years; age range 19-67 years) and 19 women (mean age, 39.9 years; age range 1988 years). Based on histopathological proof, 30 patients had Hodgkin's lymphoma (HL) and 14 was Non-Hodgkin's lymphoma (NHL).

\section{PET features and statistical analysis}

Median pretreatment tumor MTV was $147.5 \mathrm{~cm}^{3}$ (interquartile range [IQR], 89-179.2), and median pretreatment SUVmax was 23.1 (IQR, 14.6-29.1). The results of correlation of PET texture indices with the conventional quantitative metrics namely maximum SUV (SUVmax), mean SUV (SUVmean), tumor lesion glycolysis (TLG), metabolic tumor volume (MTV), SUVpeak and surface area are summarized in tables I and II in supplement material.

A general observation was that texture features obtained from all bin size analyzed have shown a moderate to strong correlation with conventional PET metrics. Spearman's rank correlation coefficients (rho) has ranged from 0.50 to 0.97 for positive correlation and ranged from -0.52 to -0.97 for the inverse correlation.

A heat map depicting the absolute value of Pearson's correlation coefficients between all pairs of textural PET features is shown in Figure 1. Correlation exceeded 0.8 (strong correlation coefficient) for $23.3 \%$ of feature pairs (17 variables in between 73 features) and exceeded 0.5 (moderate correlation coefficient) for $19.2 \%$ of feature pairs (14 variables in between 73 features). Many features were highly correlated with TLG such as contrast, entropy, homogeneity, dissimilarity, inverse difference moment and MTV; on another hand, MTV was highly correlated with features of contrast, entropy, homogeneity, dissimilarity, inverse difference moment, intensity variability, run-length variability and TLG; also SUVmax was highly correlated with mean SUV, SUV variance and SUV SD.

The AUC from ROC analysis was determined for every texture index. Out of the complete 73 textural features derived from CGITA, it was found that 8 textural features had AUC significantly higher than $0.6(p<0.05)$ for bin size 4, 8, 32 and 64 while data derived from 16 bin have not contained any significant results. 


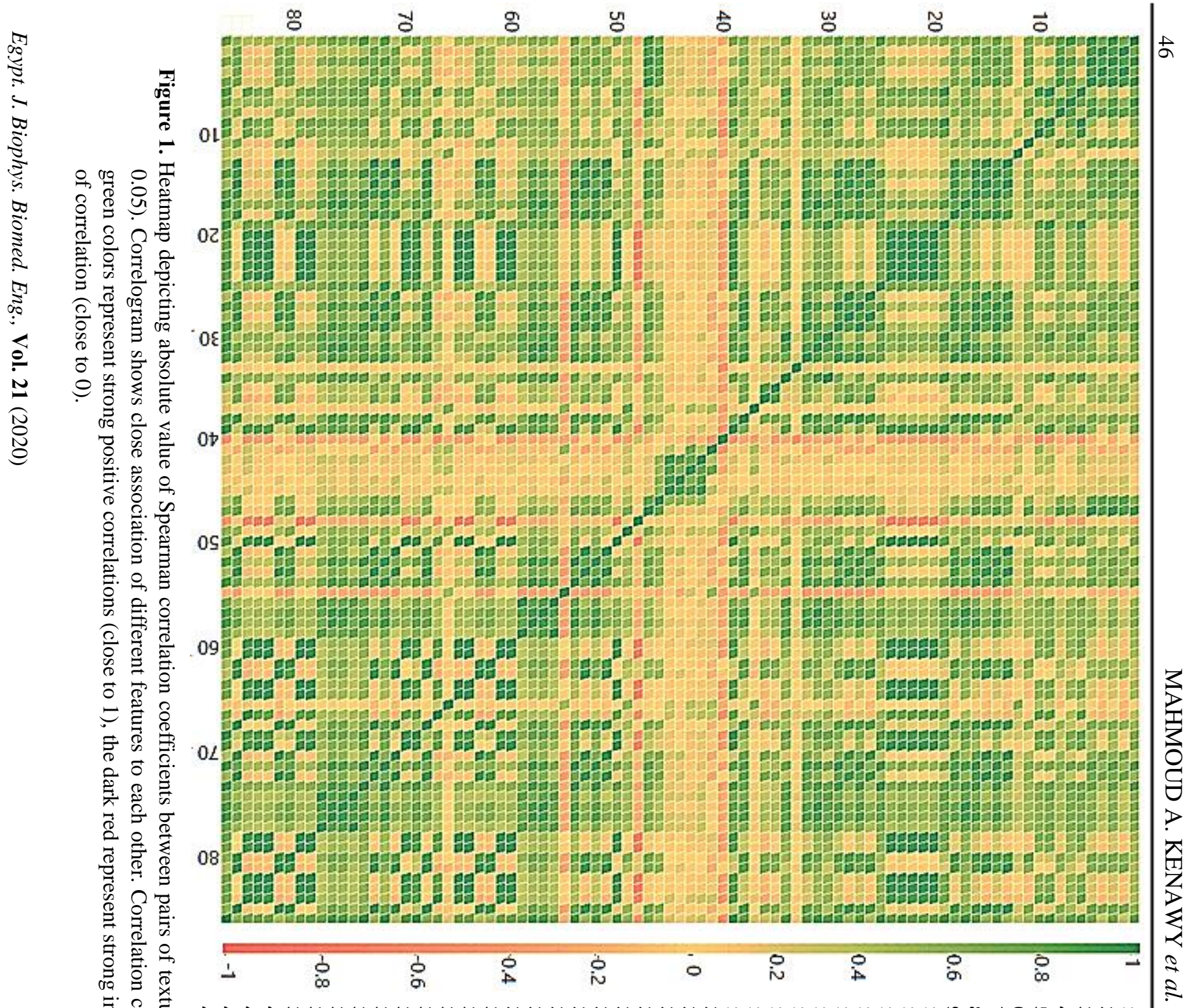

$\vec{\circ}$

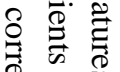

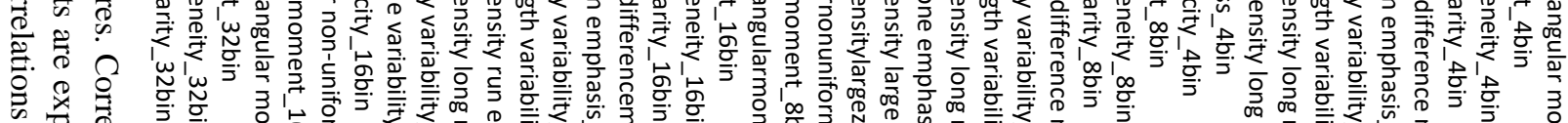

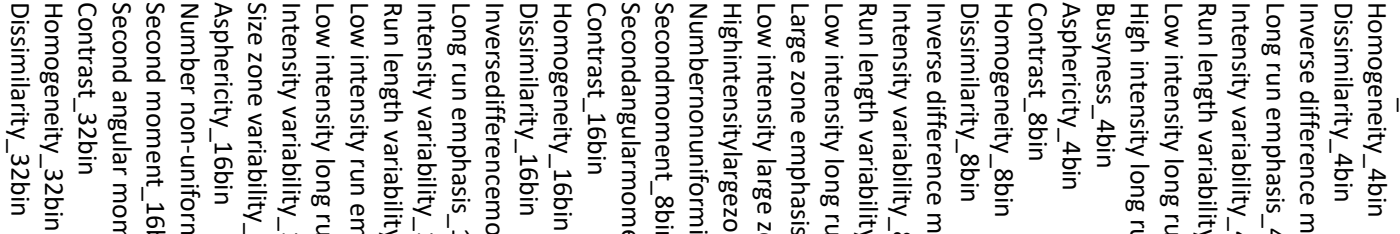



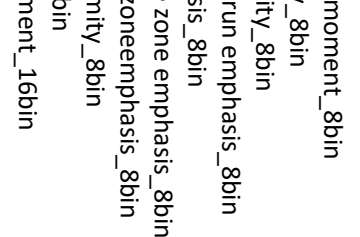

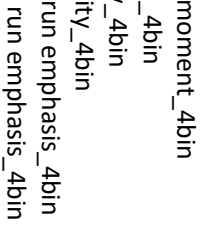

$\overrightarrow{0}$

$\stackrel{\overbrace{}}{9}$

के

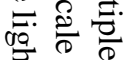

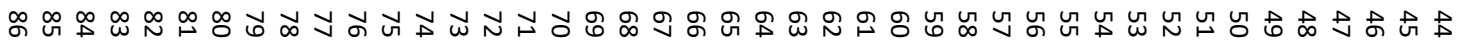

官

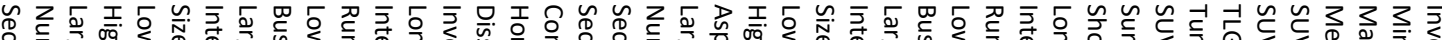

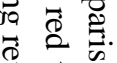

क्षे

要要

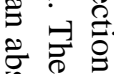

营

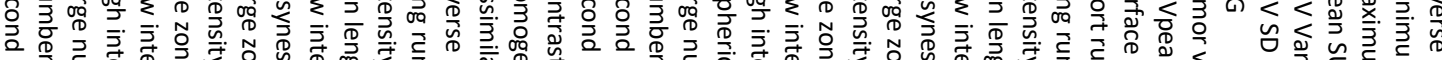

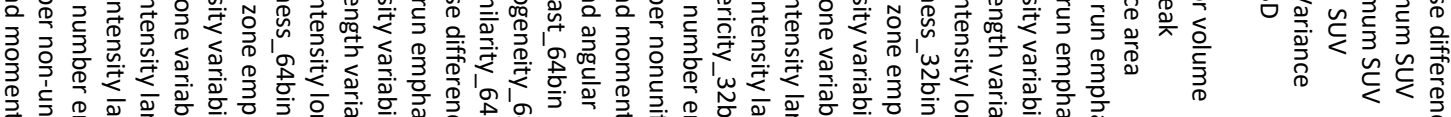

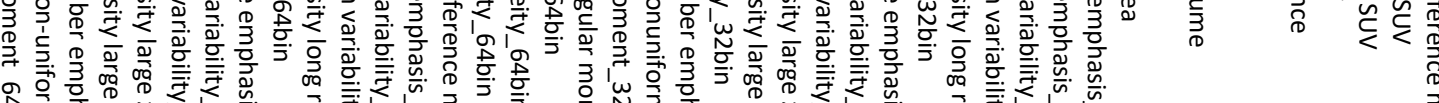

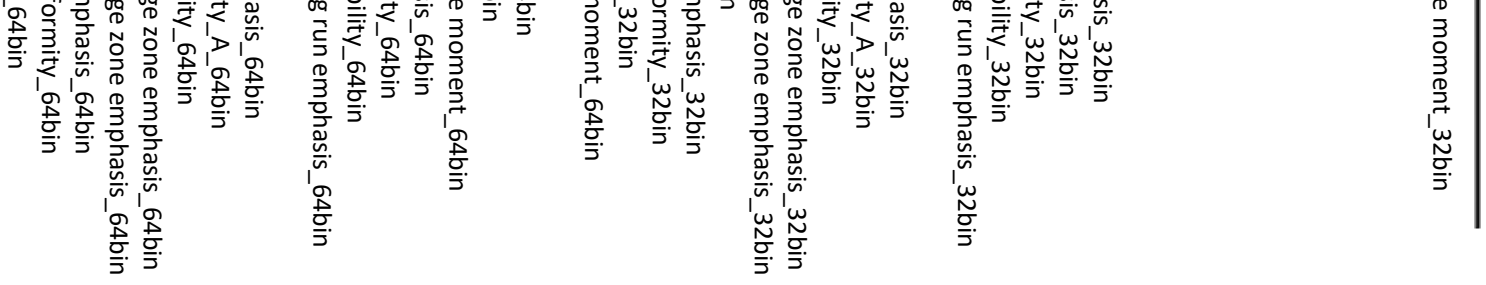


Table 2. Sensitivity, specificity and area under the curve (AUC) results of receiver operating characteristics (ROC) demonstrated for those parameters that showed a statistically significant area greater than 0.5 .

\begin{tabular}{|c|c|c|c|c|c|c|c|c|}
\hline Variable & $\begin{array}{c}\text { Bin } \\
\text { size }\end{array}$ & Sens. & Spec. & Criterion & AUC & $\begin{array}{c}\text { Lower } \\
\text { Bound }\end{array}$ & $\begin{array}{c}\text { Upper } \\
\text { Bound }\end{array}$ & Sig. \\
\hline $\begin{array}{c}\text { High-intensity long-run } \\
\text { emphasis }\end{array}$ & 4 & 66.7 & 76.9 & $>74.23$ & 0.739 & 0.587 & 0.891 & 0.008 \\
\hline $\begin{array}{c}\text { High-intensity large- } \\
\text { zone emphasis }\end{array}$ & 64 & 55.6 & 84.6 & $>140111.2$ & 0.718 & 0.563 & 0.873 & 0.015 \\
\hline Code Similarity & 32 & 55.6 & 84.6 & $\leq 0.005$ & 0.714 & 0.553 & 0.874 & 0.017 \\
\hline Max spectrum & 8 & 44.4 & 88.5 & $>0.126$ & 0.682 & 0.522 & 0.842 & 0.042 \\
\hline Long run emphasis & 64 & 61.1 & 76.9 & $>39.36$ & 0.709 & 0.554 & 0.865 & 0.019 \\
\hline Code Similarity & 64 & 50 & 88.5 & $\leq 0.008$ & 0.707 & 0.548 & 0.867 & 0.021 \\
\hline Busyness & 64 & 77.8 & 65.4 & $>2.262$ & 0.705 & 0.535 & 0.875 & 0.022 \\
\hline Large-zone emphasis & 64 & 61.1 & 76.9 & $>99688.3$ & 0.701 & 0.541 & 0.861 & 0.025 \\
\hline
\end{tabular}

Table 2 describes the texture features that showed significant AUC higher than $0.6(p<0.05)$. The ROC curves of the significant data are shown in figure 2. Along all significant data, code similarity had higher AUC with sensitivity of $(55.6 \%$ and $50 \%)$ and specificity of $(84.6 \%$ and $88.5 \%)$ for 32 and 64 bin respectively.

\section{Univariate and Multivariate binary logistic regression (UVA and MVA)}

Univariate binary logistic regression analysis performed for those features of high AUC derived from ROC data plots then to attest whether the above factors, which had prognostic values for survivals in univariate analysis would have independent prognostic significances, multivariate analysis was conducted. The results were recorded in Table 3. In univariate binary logistic analysis 2 textural features showed statistically significant association with bone infiltration whereas multivariate analysis yielded one texture feature (LRE (64 bin), $p=0.031$; OR 1.022; 95\% CI, 1.002-1.043) showed statistically significant association with bone infiltration. The set of variables in the MVA included all the significant variables in the univariate setting, using forward likelihood method.

\section{Discussion}

The tumor is composed of a heterogeneous cell population rather than a homogeneous one, with distinct molecular and phenotypic characteristics ${ }^{20}$. Biological intra-tumoral heterogeneity is may be the main reason for resistance to treatment ${ }^{21}$. Imagebased assessment of metabolic intra-tumoral heterogeneity is based on the hypothesis that it may be a projection of underlying tumor biology, including glucose metabolism, necrosis, oxygenation, vascularization and angiogenesis ${ }^{22}$. With the heightened interest in measurement of metabolic intra-tumoral heterogeneity by texture analysis, a number of clinical studies have reported that texture features from PET images have more prognostic ability than conventional SUV parameters in various cancers ${ }^{23-25}$. Although, researchers cannot interpret texture features in an intuitive way, because texture features merely offer a physical and mathematical explanation of images that can be interpreted as not only heterogeneous, but also smooth, coarse, rough, or grainy ${ }^{26}$.

Further, it has not been easy to find a consensus regarding the parameter that best represents intratumoral heterogeneity. Therefore, an integrated radiomics approach that departs from the traditional approach is required.

As it was explained in previously, quantitative features are descriptors extracted from the images using computational mathematical algorithms. The extracted features from PET/CT images using different matrices include the gray-level cooccurrence matrix (GLCM), the gray level run length matrix (GLRLM), the neighborhood intensity difference matrix (NIDM), neighborhood gray-level dependence matrix (NGLDM), and the intensity size-zone matrix (ISZM) ${ }^{12,27}$.

The hypothesis of this study was to find a correlation between tumor texture heterogeneity as revealed by radiomics analysis of primary lymphomatous lesion and bone marrow involvement. The use of texture features in differentiating between tumor cells of low or high metastatic spread could be a key tool in bone marrow involvement or at least serve as an adjunct in improving the diagnostic performance of ${ }^{18} \mathrm{~F}$ FDG PET/CT in staging and management of lymphoma patients. ${ }^{18} \mathrm{~F}-\mathrm{FDG}$ PET/CT plays an important role in diagnosis, staging, treatment monitoring, prognosis and assessment of lymphoma 28. Also, a heterogeneity metric can only have complementary (or higher significant association) value if it is not highly correlated with the corresponding volume ${ }^{29}$. The correlation between heterogeneity metrics and the conventional PET indices showed significant association for twentyone parameters (rho $\geq 0.7$ ) which have strong correl- 
Table 3. Univariate and Multivariate binary logistic regression analysis performed for those features of high AUC derived from ROC data plots.

\begin{tabular}{|c|c|c|c|c|}
\hline \multicolumn{5}{|c|}{ Univariate analysis } \\
\hline \multirow{2}{*}{ Risk factors } & \multirow{2}{*}{$p$-value } & \multirow{2}{*}{ Odds Ratio } & \multicolumn{2}{|c|}{$(95 \% \mathrm{CI})$} \\
\hline & & & Lower Bound & Jpper Bound \\
\hline Busyness (64 bin) & 0.590 & 1.011 & 0.971 & 1.052 \\
\hline Large-zone emphasis (64 bin) & 0.362 & 1.000 & 1.000 & 1.000 \\
\hline High-intensity large-zone emphasis (64 bin) & 0.340 & 1.000 & 1.000 & 1.000 \\
\hline Max spectrum $(8$ bin $)$ & 0.143 & 28.45 & 0.32 & 2525.05 \\
\hline High-intensity long-run emphasis (4 bin) & 0.102 & 1.010 & 0.991 & 1.023 \\
\hline Code Similarity (32 bin) & 0.100 & 1.000 & 1.000 & 1.000 \\
\hline Code Similarity (64 bin) & $0.039 *$ & 1.000 & 1.000 & 1.000 \\
\hline Long run emphasis (64 bin) & $0.025^{*}$ & 1.021 & 1.043 & 1.043 \\
\hline \multicolumn{5}{|c|}{ Itivariate analysis } \\
\hline Long run emphasis (64 bin) & $0.031 *$ & 1.022 & 1.002 & 1.043 \\
\hline
\end{tabular}

* Significant at type I error of 5\%. CI = confidence interval.

ation coefficient and sixteen parameters with moderate correlation $(0.7>$ rho $\geq 0.5)$. Bone marrow biopsy has been the conventional method of assessment of bone marrow infiltration. It has several staging, treatment and prognostic implications ${ }^{30}$. Limitation of bone marrow biopsy include site-dependence, discordance in morphology between lymphomatous cells in extramedullary sites and those cells in the bone marrow ${ }^{31}$. In addition, histologic data may report a different pathologic non-lymphoma related malignancy or disorder as well as its invasive nature, pain and clinician expertise. Other drawbacks include extra medical staff, sample fixation and decalcification besides more stay in the clinic. Not more likely but also add some burden to the examination is the addition of trephine biopsy with bone marrow aspiration that may cause some adverse events including hemorrhage and morbidity 32. Another major shortcoming is the likelihood of missing focal disease as biopsy normally doesn't cover the entire portion of bone marrow. These shortcomings of bone marrow biopsy are stimulating to find out alternative methods that obviate the need for such procedures while able to provide at least equivalent if not better diagnostic performance. However, ${ }^{18} \mathrm{~F}-\mathrm{FDG}$ PET/CT has several advantages in this context including full body coverage, less invasive, precise local assessment of diffuse versus focal infiltration using anatomical features as well as possibility of quantitative data interpretation. The current study focuses on the later advantage using not only the conventional PET metrics but also incorporates the

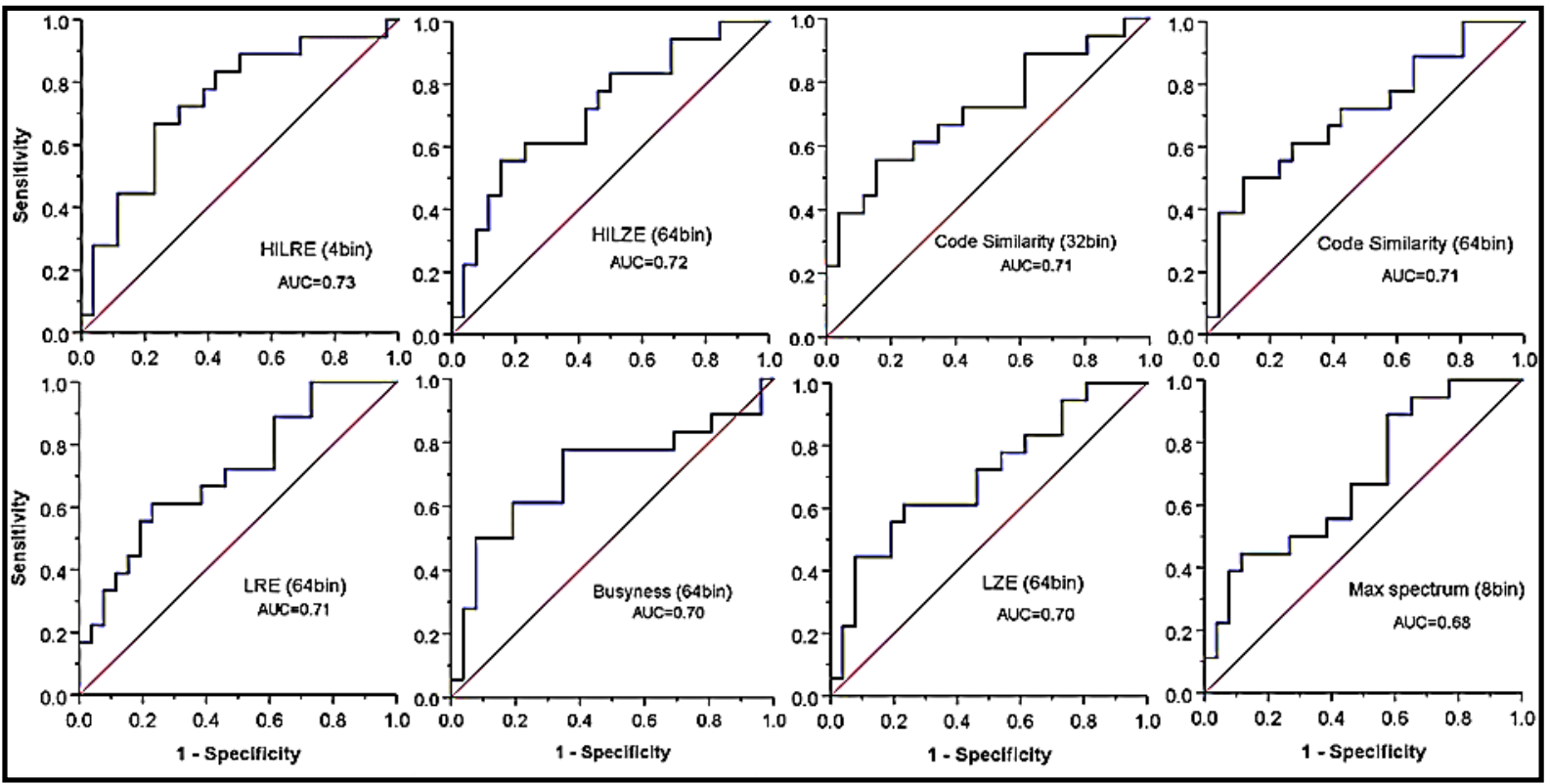

Figure 2. ROC curves of the PET heterogeneity indices. It was observed that the texture features had higher discriminative power than the conventional PET metrics. 
new advances in texture feature analysis or radiomics data extraction.

The replacement of ${ }^{18} \mathrm{~F}-\mathrm{FDG} \mathrm{PET} / \mathrm{CT}$ to bone marrow biopsy is still a matter of active debate. However, the quantitative capacity and recent advances in radiomics data analysis open up new avenues in this context. The present paper has been designed to find out the major PET feature that could be utilized in future studies as benchmark or evident source in eliciting more biological characteristics of bone marrow invasion. Several researches have confirmed a relation between greater FDG uptake and more aggressive course of malignancy in lymphoma 33,34 .

The resulting significant features confirmed that ${ }^{18}$ F-FDG PET images have advantages of combining practical and anatomical information which is regarded as contemporary trendy reference for the management of lymphomas ${ }^{35}$. In other study, when looking into the prognostic performance of the texture data, MTV was found the most statistically significant parameters that have shown the largest correlation coefficient ${ }^{29}$. A recent report looking at the differences that arise from tumor volume segmentation has shown that low MTV had a significantly longer progression free survival and overall survival compared to patients with high MTV. These findings were obtained regardless of the segmentation methods used ${ }^{36}$.

The conventional PET indices (also extracted features calculated from SUV) in calculation depends on radioactivity concentration measured by the PET scanner within a region of interest (ROI) $\left({ }^{18} \mathrm{~F}-\mathrm{FDG}\right.$ uptake interior contoured lesion) ${ }^{37}$, and biopsy (i.e. pathological sample) is the gold standard for determining tumor genetics ${ }^{38}$; also genomic features of gene expression and pathways can be predicted by FDG uptake features from the lesion ${ }^{39}$.

Therefore, the correlation between conventional matrix (such as SUV mean, SUV max, TLG) and quantitative features from PET images (especially those with strong correlation) can be considered a positive result to replace these features instead of guided biopsy to prevent delaying diagnosis and other associated complications described earlier. While ROC analysis could provide a static assessment of risk identification through cut-off points that maximize sensitivity and specificity, it can provide important information about diagnostic test performance ${ }^{40,41}$.

In this work, the event in ROC curve depends on the presence or absence of bone marrow infiltration for every individual patient. By comparing AUC of ROC curves of texture parameters, we found that texture parameters presented could have strong discriminating capabilities in identifying patients who have bone marrow infiltration. This effective diagnostic capacity was statistically absent in the conventional and standard method of quantitation including SUVmax, SUVmean, SUVpeaks, TLG and MTV.

Also in between significant parameters, highintensity large zone emphasis (HILRE) achieved the highest AUC (0.718, CI 0.563-0.873)) among all significant data of ROC curves. Long run emphasis, however, has a significant strong correlation with metabolic volume which might underscore its performance in bone marrow involvement. ${ }^{18} \mathrm{~F}-\mathrm{FDG}$ PET/CT has been widely used for lymphoma diagnosis as the specificity and sensitivity have higher indications in lymphoma regions especially in the initial staging procedures ${ }^{11,42}$.

In fact, second order and high-order statistics (i.e., based on gray-level matrix, nearest neighbor spatial dependence matrices, voxel-alignment matrix, intensity size-zone matrix, texture feature coding cooccurrence matrix, etc.) provide information from the spatial relationship of image voxels. Moreover, a multitude features may be derived from the graylevel matrices to characterize the structure of interest ${ }^{43}$. These features are independent of tumor position, orientation, size, and brightness and consider the local intensity-spatial distribution ${ }^{44}$.

Based on the ROC curves, the results showed that high-intensity long-run emphasis (HILRE 4bin) long-run emphasis (LRE 64 bin) (i.e. voxelalignment matrix ${ }^{45}$ ), high-intensity large-zone emphasis (HILZE 64 bin), large-zone emphasis (LZE 64 bin) (i.e. intensity size-zone matrix ${ }^{46}$ ), $\max$ spectrum ( 8 bin) (i.e. spectrum matrix) ${ }^{47}$, busyness (64 bin) (neighborhood gray intensity difference matrix ${ }^{48}$ ), code similarity (32 bin and 64 bin) (coding co-occurrence matrix ${ }^{49}$ ) were significant predictors of bone marrow infiltration $(\mathrm{p}<0.05)$. These features were extracted from the second and higher-order matrix, highlighting the relevance of voxel arrangement and their spatial correlation to the underlying biological processes of bone marrow infiltration.

To our knowledge, this finding has not been reported in the literature. Busyness extracted from contoured PET images offered a greater discriminatory power marked by higher accuracy, specificity, and sensitivity than commonly used quantitative metrics such as SUVmax which was not significant in this study. It has been reported that the NGIDM texture features was able to differentiate malignant pulmonary nodules ${ }^{50}$. The enhancement in discriminatory performance shown in this study could benefit patients by preventing the high falsepositive rate of PET for FDG uptake regions.

One limitation of the present study was the pathological guidance of bone marrow infiltration in the selected patient cohort. This should be tackled in validation studies investigating the role of texture feature analysis in diagnosing bone marrow infiltration in lymphoma patients. Future studies are therefore highly warranted to confirm the 
performance of the texture data described here with special emphasis placed on code similarity and long run emphasis.

\section{Conclusion}

In conclusion, this study confirmed that FDG PET textural parameters have an effective role to differentiate lymphoma disease with bone marrow infiltration so, study proposed a new method for differentiating lymphoma disease. More investigations are highly warranted to combine the role of texture analysis in providing additional diagnostic as well as prognostic values to ${ }^{18} \mathrm{~F}-\mathrm{FDG}$ $\mathrm{PET} / \mathrm{CT}$ in initial staging of patient with lymphoma.

\section{References}

1. D'Souza, M. M.; Jaimini, A.; Bansal, A., et al. FDG$\mathrm{PET} / \mathrm{CT}$ in lymphoma, Indian $J$ Radiol Imaging. 2013, 23, 354-365.

2. Toma, P.; Granata, C.; Rossi, A.; Garaventa, A. Multimodality imaging of Hodgkin disease and non-Hodgkin lymphomas in children, Radiographics : a review publication of the Radiological Society of North America, Inc. 2007, 27, 1335-1354.

3. Armitage, J. O. Staging non-Hodgkin lymphoma, CA: a cancer journal for clinicians. 2005, 55, 368376.

4. Vinnicombe, S. J.; Reznek, R. H. Computerised tomography in the staging of Hodgkin's disease and non-Hodgkin's lymphoma, European journal of nuclear medicine and molecular imaging. 2003, 30 Suppl 1, S42-55.

5. Dhanapathi, H.; Kumar, R. J. I. J. M. P. O. F-18 FDG $\mathrm{PET} / \mathrm{PET}-\mathrm{CT}$ in the management of lymphoma. 2007, 28, 17-23.

6. Lister, T. A.; Crowther, D.; Sutcliffe, S. B., et al. Report of a committee convened to discuss the evaluation and staging of patients with Hodgkin's disease: Cotswolds meeting, Journal of clinical oncology : official journal of the American Society of Clinical Oncology. 1989, 7, 1630-1636.

7. Weber, A. L.; Rahemtullah, A.; Ferry, J. A. Hodgkin and non-Hodgkin lymphoma of the head and neck: clinical, pathologic, and imaging evaluation, Neuroimaging clinics of North America. 2003, 13, 371-392.

8. Carr, R.; Barrington, S.; Madan, B., et al. Detection of LYMPHOMA in bone marrow by whole-body positron emission tomography, Blood. 1998, 91, 3340-3346.
9. Ngeow, J. Y. Y.; Quek, R. H. H.; Ng, D. C. E., et al. High SUV uptake on FDG-PET/CT predicts for an aggressive B-cell lymphoma in a prospective study of primary FDG-PET/CT staging in lymphoma, Annals of oncology : official journal of the European Society for Medical Oncology. 2009, 20, 1543-1547.

10. Pelosi, E.; Penna, D.; Douroukas, A., et al. Bone marrow disease detection with FDG-PET/CT and bone marrow biopsy during the staging of malignant lymphoma: results from a large multicentre study, The quarterly journal of nuclear medicine and molecular imaging : official publication of the Italian Association of Nuclear Medicine (AIMN) [and] the International Association of Radiopharmacology (IAR), [and] Section of the So. 2011, 55, 469-475.

11. Paes, F. M.; Kalkanis, D. G.; Sideras, P. A.; Serafini, A. N. FDG PET/CT of extranodal involvement in non-Hodgkin lymphoma and Hodgkin disease, Radiographics. 2010, 30, 269-291.

12. Fang, Y. H.; Lin, C. Y.; Shih, M. J., et al. Development and evaluation of an open-source software package "CGITA" for quantifying tumor heterogeneity with molecular images, BioMed research international. 2014, 2014, 248505.

13. Cheng, N. M.; Fang, Y. H.; Chang, J. T., et al. Textural features of pretreatment 18F-FDG $\mathrm{PET} / \mathrm{CT}$ images: prognostic significance in patients with advanced T-stage oropharyngeal squamous cell carcinoma, Journal of nuclear medicine : official publication, Society of Nuclear Medicine. 2013, 54, 1703-1709.

14. Wahl, R. L.; Jacene, H.; Kasamon, Y.; Lodge, M. A. From RECIST to PERCIST: Evolving Considerations for PET response criteria in solid tumors, J Nucl Med. 2009, 50 Suppl 1, 122s-150s.

15. Castellano, G.; Bonilha, L.; Li, L. M.; Cendes, F. Texture analysis of medical images, Clin Radiol. 2004, 59, 1061-1069.

16. Soh, L. K.; Tsatsoulis, C. Texture analysis of SAR sea ice imagery using gray level co-occurrence matrices, IEEE Transactions on Geoscience and Remote Sensing. 1999, 37, 780-795.

17. Lam, S. W. Texture feature extraction using gray level gradient based co-occurence matrices, Proccedings of Conference Texture feature extraction using gray level gradient based cooccurence matrices, 1996, 1, 267-271 vol.261.

18. Sultan, S.; Baloch, N.; Ahmed, Z. A.; Irfan, S. M.; Parveen, $\mathrm{S}$. Pattern of bone marrow involvement 
in non-Hodgkin's lymphoma classified according to WHO classification: Report of a developing country Pakistan, J Lab Physicians. 2018, 10, 1720.

19. Xu, H.; Guo, W.; Cui, X., et al. Three-Dimensional Texture Analysis Based on PET/CT Images to Distinguish Hepatocellular Carcinoma and Hepatic Lymphoma, Front Oncol. 2019, 9, 844844.

20.Zhang, J.; Fujimoto, J.; Zhang, J., et al. Intratumor heterogeneity in localized lung adenocarcinomas delineated by multiregion sequencing, Science. 2014, 346, 256-259.

21.Gerlinger, M.; Swanton, C. How Darwinian models inform therapeutic failure initiated by clonal heterogeneity in cancer medicine, British journal of cancer. 2010, 103, 1139-1143.

22. Basu, S.; Kwee, T. C.; Gatenby, R.; Saboury, B.; Torigian, D. A.; Alavi, A. Evolving role of molecular imaging with PET in detecting and characterizing heterogeneity of cancer tissue at the primary and metastatic sites, a plausible explanation for failed attempts to cure malignant disorders, European journal of nuclear medicine and molecular imaging. 2011, 38, 987-991.

23. Chung, H. H.; Kang, S. Y.; Ha, S., et al. Prognostic value of preoperative intratumoral FDG uptake heterogeneity in early stage uterine cervical cancer, Journal of gynecologic oncology. 2016, 27, e15.

24. Cheng, N. M.; Fang, Y. H.; Lee, L. Y., et al. Zonesize nonuniformity of $18 \mathrm{~F}-\mathrm{FDG}$ PET regional textural features predicts survival in patients with oropharyngeal cancer, European journal of nuclear medicine and molecular imaging. 2015, 42, 419-428.

25.Schürch, C. M.; Federmann, B.; QuintanillaMartinez, L.; Fend, F. Tumor Heterogeneity in Lymphomas: A Different Breed, Pathobiology : journal of immunopathology, molecular and cellular biology. 2018, 85, 130-145.

26. Castellano, G.; Bonilha, L.; Li, L. M.; Cendes, F. Texture analysis of medical images, Clinical Radiology. 2004, 59, 1061-1069.

27. Larue, R.; van Timmeren, J. E.; de Jong, E. E. C., et al. Influence of gray level discretization on radiomic feature stability for different $\mathrm{CT}$ scanners, tube currents and slice thicknesses: a comprehensive phantom study, Acta Oncol. 2017, 56, 1544-1553.
28. Chen, Y.; Zhou, M.; Liu, J.; Huang, G. Prognostic Value of Bone Marrow FDG Uptake Pattern of PET/CT in Newly Diagnosed Diffuse Large B-cell Lymphoma, Journal of Cancer. 2018, 9, 12311238.

29. Hatt, M.; Tixier, F.; Pierce, L.; Kinahan, P. E.; Le Rest, C. C.; Visvikis, D. Characterization of PET/CT images using texture analysis: the past, the present... any future?, European journal of nuclear medicine and molecular imaging. 2017, 44, 151-165.

30. Adams, H. J.; Nievelstein, R. A.; Kwee, T. C. Opportunities and limitations of bone marrow biopsy and bone marrow FDG-PET in lymphoma, Blood Rev. 2015, 29, 417-425.

31. Arber, D. A.; George, T. I. Bone marrow biopsy involvement by non-Hodgkin's lymphoma: frequency of lymphoma types, patterns, blood involvement, and discordance with other sites in 450 specimens, The American journal of surgical pathology. 2005, 29, 1549-1557.

32. Bain, B. J. Morbidity associated with bone marrow aspiration and trephine biopsy - a review of UK data for 2004, Haematologica. 2006, 91, 1293-1294.

33. Schoder, H.; Noy, A.; Gonen, M., et al. Intensity of 18fluorodeoxyglucose uptake in positron emission tomography distinguishes between indolent and aggressive non-Hodgkin's lymphoma, Journal of clinical oncology : official journal of the American Society of Clinical Oncology. 2005, 23, 4643-4651.

34. Wu, X.; Pertovaara, H.; Korkola, P., et al. Correlations between functional imaging markers derived from PET/CT and diffusionweighted MRI in diffuse large B-cell lymphoma and follicular lymphoma, PloS one. 2014, 9, e84999-e84999.

35. Okada, M.; Sato, N.; Ishii, K.; Matsumura, K.; Hosono, M.; Murakami, T. FDG PET/CT versus CT, MR Imaging, and 67Ga Scintigraphy in the Posttherapy Evaluation of Malignant Lymphoma, RadioGraphics. 2010, 30, 939-957.

36. Ceriani, L.; Milan, L.; Johnson, P. W. M., et al. Baseline PET features to predict prognosis in primary mediastinal B cell lymphoma: a comparative analysis of different methods for measuring baseline metabolic tumour volume, European journal of nuclear medicine and molecular imaging. 2019, 46, 1334-1344. 
37. Lucignani, G.; Paganelli, G.; Bombardieri, E. The use of standardized uptake values for assessing FDG uptake with PET in oncology: a clinical perspective, Nuclear medicine communications. 2004, 25, 651-656.

38. Moon, S. H.; Kim, J.; Joung, J.-G., et al. Correlations between metabolic texture features, genetic heterogeneity, and mutation burden in patients with lung cancer, European journal of nuclear medicine and molecular imaging. 2019, 46, 446-454.

39. Ahn, K. S.; Kang, K. J.; Kim, Y. H., et al. Genetic features associated with (18)F-FDG uptake in intrahepatic cholangiocarcinoma, Ann Surg Treat Res. 2019, 96, 153-161.

40. Fan, J.; Upadhye, S.; Worster, A. Understanding receiver operating characteristic (ROC) curves, CJEM. 2006, 8, 19-20.

41. Kamarudin, A. N.; Cox, T.; Kolamunnage-Dona, R. Time-dependent ROC curve analysis in medical research: current methods and applications, $B M C$ Med Res Methodol. 2017, 17, 53-53.

42. Kwee, T. C.; Kwee, R. M.; Nievelstein, R. A. Imaging in staging of malignant lymphoma: a systematic review, Blood. 2008, 111, 504-516.

43. El Naqa, I.; Grigsby, P.; Apte, A., et al. Exploring feature-based approaches in PET images for predicting cancer treatment outcomes, Pattern recognition. 2009, 42, 1162-1171.
44. Greven, K. M. Positron-emission tomography for head and neck cancer, Seminars in radiation oncology. 2004, 14, 121-129.

45. Loh, H.; Leu, J.; Luo, R. C. The analysis of natural textures using run length features, IEEE Transactions on Industrial Electronics. 1988, 35, 323-328.

46. Thibault, G.; Fertil, B.; Navarro, C., et al. Texture Indexes and Gray Level Size Zone Matrix Application to Cell Nuclei Classification, 2009.

47. He, D.-C.; Wang, L. Texture features based on texture spectrum, Pattern recognition. 1991, 24, 391-399.

48. Amadasun, M.; King, R. Textural features corresponding to textural properties, IEEE Transactions on Systems, Man, and Cybernetics. 1989, 19, 1264-1274.

49. Horng, M. H.; Sun, Y. N.; Lin, X. Z. Texture feature coding method for classification of liver sonography, Computerized medical imaging and graphics : the official journal of the Computerized Medical Imaging Society. 2002, 26, 33-42.

50. Chen, S.; Harmon, S.; Perk, T., et al. Using neighborhood gray tone difference matrix texture features on dual time point PET/CT images to differentiate malignant from benign FDG-avid solitary pulmonary nodules, Cancer imaging : the official publication of the International Cancer Imaging Society. 2019, 19, 56-56. 


\section{Supplementary Material}

Table S.I. Summary of the texture feature extracted from CGITA package.

\begin{tabular}{|c|c|}
\hline Parent matrix & Feature measure \\
\hline Co-occurrence matrix [4] & $\begin{array}{l}\text { Second angular moment, contrast, entropy, homogeneity, } \\
\text { dissimilarity, inverse difference moment. }\end{array}$ \\
\hline Voxel-alignment matrix [5] & $\begin{array}{l}\text { Short-run emphasis, long-run emphasis, intensity variability, } \\
\text { run-length variability, run percentage, low-intensity run } \\
\text { emphasis, high-intensity run emphasis, low-intensity short- } \\
\text { run emphasis, high-intensity short-run emphasis, low- } \\
\text { intensity long-run emphasis, high-intensity long-run } \\
\text { emphasis. }\end{array}$ \\
\hline $\begin{array}{l}\text { Neighborhood intensity } \\
\text { difference matrix [6] }\end{array}$ & Coarseness, contrast, busyness, complexity, strength. \\
\hline Intensity size-zone matrix [7] & $\begin{array}{l}\text { Short-zone emphasis, large-zone emphasis, intensity } \\
\text { variability, size-zone variability, zone percentage, low- } \\
\text { intensity zone emphasis, high-intensity zone emphasis, low- } \\
\text { intensity short-zone emphasis, high-intensity short-zone } \\
\text { emphasis, low-intensity large-zone emphasis, high-intensity } \\
\text { large-zone emphasis. }\end{array}$ \\
\hline $\begin{array}{l}\text { Normalized co-occurrence } \\
\text { matrix [4] }\end{array}$ & $\begin{array}{l}\text { Second angular moment, contrast, entropy, homogeneity, } \\
\text { inverse difference moment, dissimilarity, correlation. }\end{array}$ \\
\hline Voxel statistics & $\begin{array}{l}\text { Minimum SUV, maximum SUV, mean SUV, SUV variance, SUV } \\
\text { SD, SUV skewness, SUV kurtosis, SUV skewness (bias } \\
\text { corrected), SUV kurtosis (bias corrected), TLG, tumor volume, } \\
\text { entropy, SULpeak }\end{array}$ \\
\hline Texture spectrum [8] & Max spectrum, Black-white symmetry. \\
\hline Texture feature coding [9] & $\begin{array}{l}\text { Coarseness, homogeneity, mean convergence Second angular } \\
\text { moment, contrast, entropy, homogeneity, intensity, inverse } \\
\text { difference moment, correlation, variance, code similarity. }\end{array}$ \\
\hline $\begin{array}{l}\text { Texture feature coding co- } \\
\text { occurrence matrix [9] }\end{array}$ & $\begin{array}{l}\text { Second angular moment, contrast, entropy, homogeneity, } \\
\text { intensity, inverse difference moment, correlation, variance, } \\
\text { code entropy. }\end{array}$ \\
\hline $\begin{array}{l}\text { Neighborhood gray-level } \\
\text { dependence [10] }\end{array}$ & $\begin{array}{l}\text { Small-number emphasis, large-number emphasis, number } \\
\text { non-uniformity, second moment, entropy. }\end{array}$ \\
\hline
\end{tabular}


Table S.II. Spearman correlation between texture features and the most commonly used PET quantitative metrics. The table shows variable degree of associations being highest and strong correlation coefficient.

\begin{tabular}{|c|c|c|c|c|c|}
\hline \multicolumn{6}{|c|}{ Strong correlation } \\
\hline \multirow{3}{*}{ Variable } & \multicolumn{5}{|c|}{ Correlation Coefficient (Spearman's rho, $\rho$ ) } \\
\hline & \multicolumn{5}{|c|}{ TLG } \\
\hline & 4 & 8 & 16 & 32 & 64 \\
\hline Contrast & 0.829 & 0.855 & 0.854 & 0.854 & 0.855 \\
\hline Entropy & -0.798 & -0.798 & -0.780 & -0.763 & -0.727 \\
\hline Homogeneity & 0.790 & 0.784 & 0.754 & 0.731 & 0.701 \\
\hline Dissimilarity & 0.825 & 0.847 & 0.849 & 0.848 & 0.852 \\
\hline Inverse difference moment & 0.790 & 0.779 & 0.732 & 0.707 & 0.702 \\
\hline \multirow[t]{2}{*}{ Tumor volume } & 0.792 & 0.792 & 0.789 & 0.796 & 0.792 \\
\hline & \multicolumn{5}{|c|}{ Surface area } \\
\hline \multirow[t]{2}{*}{ A sphericity } & 0.798 & 0.798 & 0.797 & 0.793 & 0.798 \\
\hline & \multicolumn{5}{|c|}{ MTV } \\
\hline Contrast & 0.859 & 0.849 & 0.813 & 0.801 & 0.795 \\
\hline Entropy & -0.967 & -0.969 & -0.969 & -0.969 & -0.946 \\
\hline Homogeneity & 0.970 & 0.969 & 0.961 & 0.950 & 0.909 \\
\hline Dissimilarity & 0.862 & 0.892 & 0.893 & 0.887 & 0.888 \\
\hline Inverse difference moment & 0.970 & 0.969 & 0.954 & 0.920 & 0.827 \\
\hline Intensity variability & 0.754 & 0.742 & 0.751 & 0.772 & 0.748 \\
\hline Run-length variability & 0.839 & 0.883 & 0.865 & 0.844 & 0.811 \\
\hline \multirow[t]{2}{*}{ TLG } & 0.792 & 0.792 & 0.789 & 0.796 & 0.792 \\
\hline & \multicolumn{5}{|c|}{ SUVmean } \\
\hline Minimum SUV & 0.731 & 0.731 & 0.736 & 0.704 & 0.731 \\
\hline Maximum SUV & 0.801 & 0.801 & 0.811 & 0.756 & 0.801 \\
\hline \multirow[t]{2}{*}{ SUV SD } & 0.865 & 0.865 & 0.870 & 0.850 & 0.865 \\
\hline & \multicolumn{5}{|c|}{ SUVmax } \\
\hline Mean SUV & 0.801 & 0.801 & 0.811 & 0.756 & 0.801 \\
\hline SUV Variance & 0.836 & 0.836 & 0.796 & 0.876 & 0.836 \\
\hline SUV SD & 0.930 & 0.930 & 0.932 & 0.929 & 0.930 \\
\hline
\end{tabular}

Table II. Spearman correlation between texture features and the most commonly used PET quantitative metrics. The table shows variable degree of associations being higher and moderate correlation coefficient.

\begin{tabular}{|c|c|c|c|c|c|}
\hline \multicolumn{6}{|c|}{ Moderate correlation } \\
\hline \multirow{3}{*}{ Variable } & \multicolumn{5}{|c|}{ Correlation Coefficient (Spearman's rho, $\rho$ ) } \\
\hline & \multicolumn{5}{|c|}{ TLG } \\
\hline & 4 & 8 & 16 & 32 & 64 \\
\hline Intensity variability & 0.529 & 0.519 & 0.530 & 0.565 & 0.581 \\
\hline Run-length variability & 0.632 & 0.700 & 0.677 & 0.670 & 0.681 \\
\hline Maximum SUV & 0.530 & 0.530 & 0.528 & 0.527 & 0.530 \\
\hline Mean SUV & 0.502 & 0.502 & 0.500 & 0.502 & 0.502 \\
\hline SUV Variance & 0.583 & 0.583 & 0.579 & 0.587 & 0.583 \\
\hline \multirow[t]{2}{*}{ SUV SD } & 0.582 & 0.582 & 0.546 & 0.590 & 0.582 \\
\hline & \multicolumn{5}{|c|}{ Surface area } \\
\hline Entropy & -0.526 & -0.531 & -0.520 & -0.553 & -0.543 \\
\hline Homogeneity & 0.543 & 0.545 & 0.527 & 0.560 & 0.539 \\
\hline Inverse difference moment & 0.543 & 0.547 & 0.531 & 0.557 & 0.504 \\
\hline \multirow[t]{2}{*}{ Tumor volume } & 0.635 & 0.635 & 0.627 & 0.641 & 0.635 \\
\hline & \multicolumn{5}{|c|}{ MTV } \\
\hline Long run emphasis & 0.5 & 0.501 & 0.588 & 0.672 & 0.634 \\
\hline Low-intensity long-run emphasis & 0.5 & 0.503 & 0.593 & 0.677 & 0.636 \\
\hline \multirow[t]{2}{*}{ Surface area } & 0.635 & 0.635 & 0.627 & 0.641 & 0.635 \\
\hline & \multicolumn{5}{|c|}{ SUVmean } \\
\hline SUV Variance & 0.655 & 0.655 & 0.636 & 0.653 & 0.655 \\
\hline \multirow[t]{2}{*}{ TLG } & 0.502 & 0.502 & 0.501 & 0.502 & 0.502 \\
\hline & \multicolumn{5}{|c|}{ SUVmax } \\
\hline TLG & 0.530 & 0.530 & 0.525 & 0.527 & 0.530 \\
\hline
\end{tabular}


دور تحليل البيانات والراديومكس في التنبؤ بتسلل النخاع العظمي في مرضى سرطان الغدد الليمفاوية

محمود عبد الحافظ قناوي، مجدي محمد خليل²، محمود حسن عبد الجواد1 وهاني حمدي البهنساوي11

1. شعبة الفيزياء الحيوية - قسم الفيزياء - كلية العلوم (بنين) - جامعة الأزهر - مدينة نصر - القاهرة - مصر.

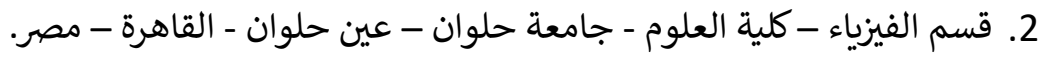

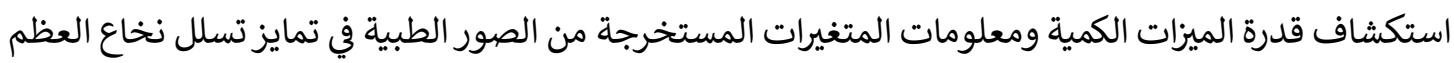

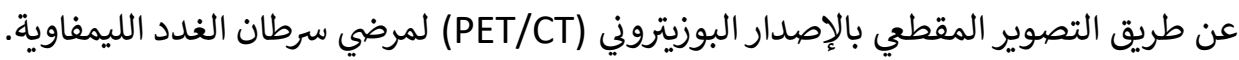

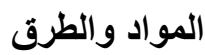

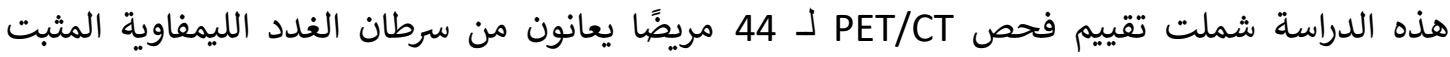

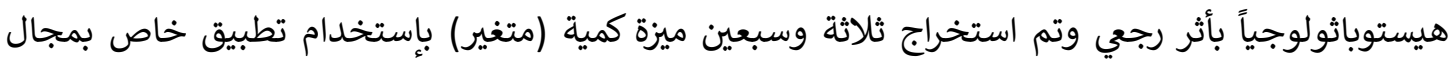

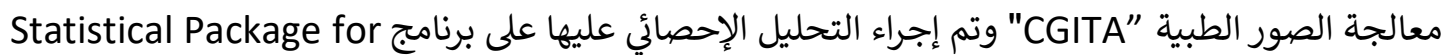
.SPSS"the Social Sciences

النتائج

أظهر تحليل الإرتباط لبعض المتغيرات المستخرجة من الصور عن وجود علاقة إيجابية طردية قوية بين

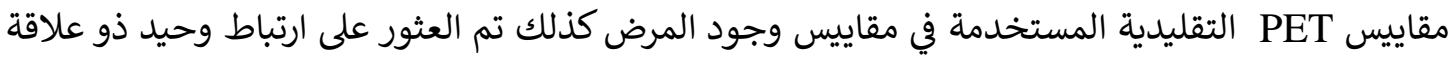

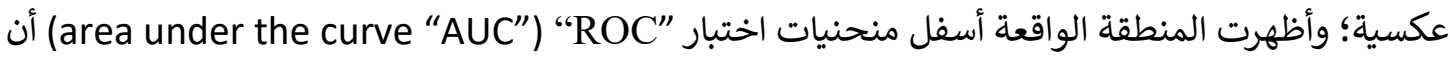
ثمانية من المتغيرات المستخرجة من صور المرضي وهي: (HILRE (4-bin), HILZE (64-bin), LRE (64-bin), LZE (64-bin), max spectrum (8-bin), busyness (64-bin), code similarity (32-bin \& 64-bin))

كانت ذات تنبؤ لتسلل نخاع العظام (AUC> 0.682) من بين الميزات الأخرى وكانت لها قيمة من 0.05؛ كما أظهرت التحليلات أحادية المتغير للمتغيرات المستخرجة أن: لهما تنبؤ كبير بتسلل المرض لنخاع العظم؛كذلك أظهرت (code similarity \& LRE (both 64 bin)) التحليلات متعددة المتغيرات أن المتغير:

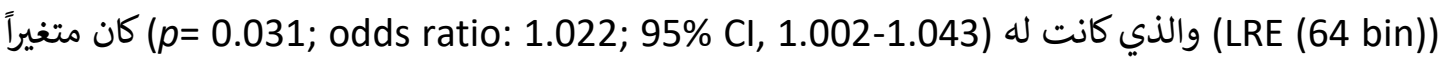
مستقلاً لتسلل نخاع العظم في سرطان الغدد الليمفاوية. الاستنتاجات يوجد ارتباطات قوية بين المتغيرات المستخرجة من صور PET وتسلل نخاع العظم في سرطان الغدد الليمفاوية

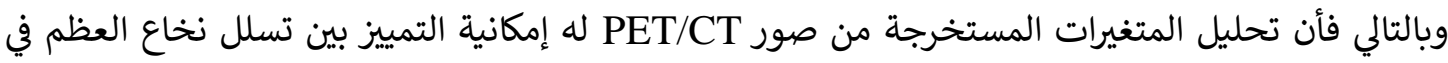

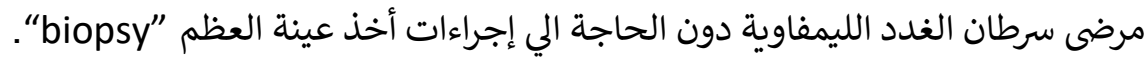

